\title{
Intellect in evolution
}

\author{
Douglas J. Futuyma
}

Evolution: Essays in Honour of John Maynard Smith. Edited by P.J. Greenwood, P.H. Harvey and M. Slatkin.

Cambridge University Press: 1985. Pp.328. £30, \$49.50.

BY the fertility and originality of his thought, the variety of his interests and contributions, and the influence of his effervescent personality on his colleagues and students, John Maynard Smith has taken his place as one of this era's leading evolutionary biologists. His empirical and theoretical contributions have included functional morphology, Drosophila genetics and cytology, the genetics of developmental patterns, the evolution of senescence, the theory of polymorphism, speciation, evolutionary rates, and kin and group selection, the evolution of sex, and the evolution of behaviour. This Festschrift in honour of Maynard Smith on his imminent retirement from Sussex University therefore marks the end of an era.

Maynard Smith, perhaps more than any other single individual, has embraced both major strategies of explanation of evolutionary change: the formal theory of population genetics, including natural selection; and the phenotypic theory of adaptations. This second theory of adaptive strategies is largely devoid of genetics, but attempts to predict, for real phenotypic characters, the ordering of fitness values that appear as abstract constructs in population genetics. Both of these approaches, between which there is considerable tension, are represented in this collection.

The power of population genetics is revealed in Slatkin's theoretical treatment of the role somatic mutation might play in defending plants against attack by creating genetic mosaics (he concludes it will usually have little effect), and by Bengtsson's review of the number of underdominant loci required to establish a sterility barrier to gene flow between incipient species (generally many are required). These essays exemplify Lewontin's point that the role of population genetic theory is not to predict evolution, but to delineate the prohibited and the possible. Lewontin is less encouraging on the ability of theory to explain observations (for example to specify causes for gene frequency distributions), but is hopeful that variation at the nucleotide level may "give a finer structure of genetic detail corresponding to the detail that is built into the theory". Yet the theory includes elements such as population size, gene flow and linkage disequilibrium that surely will never be known in such detail, so the scope for such optimism may be rather narrow.
Representing the population genetic approach to adaptations, Felsenstein provides an intriguing distillation of theories of recombination, with some surprising conclusions (for example, the sibcompetition model of Williams is a special case of Fisher and Muller's theory). Michod and Sanderson develop a theory of behavioural structure that obviates the

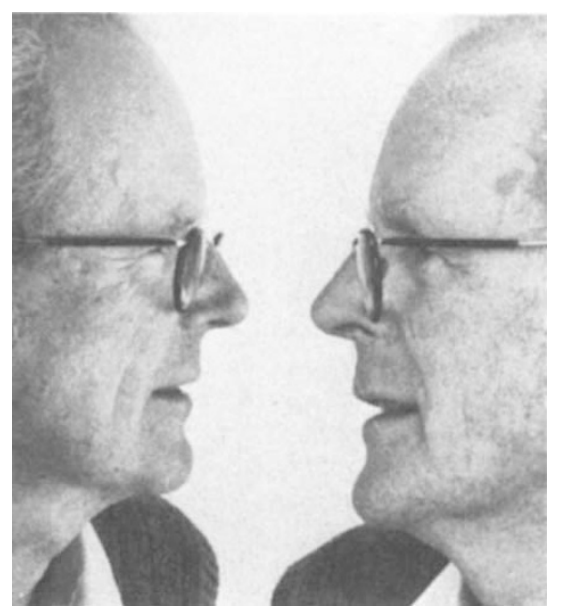

Maynard Smith: a view of both major strategies of explanation of evolutionary change.

need for kin selection as a necessary explanation of cooperation. Rose reviews his work on the genetics of senescence.

Strategic analysis has become one of the chief tools in the study of adaptations, and Maynard Smith's introduction of the evolutionary stable strategy (ESS) concept is surely one of his most important contributions. The pitfalls lie not so much in the theory, which like population genetics theory varies in the realism of its assumptions, but in the testing, especially by the comparative method. Charnov shows the power of ESS theory to predict sex ratio and related phenomena, and uses the comparative method to good advantage. Packer and Pusey make a fairly convincing case for the ability of ESS theory to predict the outcome of asymmetrical contests in social mammals.

But observations do not always conform to the theory, as both Clutton-Brock and Parker and Hammerstein note, or they may conform for the wrong reasons. For example, Silvertown cites the composite Hypochoeris glabra in support of the hypothesis that the proportion of fardispersing fruits should increase with fruit number because of density-dependent competition. But because near-dispersed fruits develop only on the circumference of the capitulum, the conformity to expectations is a necessary allometric consequence of the ratio of circumference to the area.

In other instances, the sensitivity of the test is inadequate. For example, D. Charlesworth attempts an admirably careful test of the hypothesis that dioecy has evolved in angiosperms as an outcrossing mechanism, by seeing if it is negatively correlated with self-incompatibility across families and genera. The data are inadequate for the test, but even if they were adequate, a census of this kind is not sufficient, because a phylogenetic analysis is required in each case to infer whether dioecy arose in a self-compatible or selfincompatible ancestor.

We are now all aware of the excess of overenthusiastic adaptationism, and of the difficulties of testing strategic theories of adaptation. But the theory itself may prove to have important limitations, as Parker and Hammerstein point out in an excellent review of evolutionary game theory. For example, realistic extensive games in which the current decision depends on previous events typically have multiple stable solutions. Thus the historical contingency of evolution is inescapable, even in optimality models. Parker and Hammerstein also note that the necessary synthesis of strategic theory with population genetics has not yet occurred and may prove difficult - as the schism within this book bears witness.

Among the remaining essays, some fall within these two approaches: Seger presents a very restricted genetic model of sympatric speciation, León theorizes on seed germination, Gittleman reviews possible advantages of aggregated placing of young by mammals, and Greenwood and Wheeler offer a highly speculative physiological explanation of sexual size dimorphism in homeotherms.

A potpourri of other contributions includes an exemplary exercise in adaptive hypothesis testing by Harvey and Ralls concerning body size in weasels, an intriguing failure by Bradbury, Vehrencamp and Gibson to find a mechanistic basis for the variance in male reproductive success in lek-forming species, an elaboration by Stenseth of Maynard Smith and Stenseth's theory of lag-load and evolutionary rates, and a benediction by May.

This well-edited volume offers a nicely balanced overview of some of the most active areas of investigation of adaptation. Like most honorary volumes, it is neither deep nor comprehensive, but several of the essays, as I have described, are important and thoughtful, and will surely be cited widely.

Douglas J. Futuyma is in the Department of Ecology and Evolution, State University of New York, Stony Brook, New York 11794, USA. 